\title{
Bayesian assessment of diagnostic accuracy of a commercial borescope and of trimming chute exams for diagnosing digital dermatitis in dairy cows
}

\author{
Salvatore Ferraro, ${ }^{1}$ Sébastien Buczinski, ${ }^{1 *}$ × Simon Dufour, ${ }^{2}{ }^{\oplus}$ Marjolaine Rousseau, ${ }^{1}$ Jocelyn Dubuc, ${ }^{1}$ (]) \\ Jean-Philippe Roy, ${ }^{1}$ (D) and André Desrochers ${ }^{1}$ (D) \\ ${ }^{1}$ Département de Sciences Cliniques, Faculté de Médecine Vétérinaire, Université de Montréal, 3200 rue Sicotte, St-Hyacinthe, Québec, \\ J2S 2M2, Canada \\ ${ }^{2}$ Département de Pathologie et Microbiologie, Faculté de Médecine Vétérinaire, Université de Montréal, 3200 rue Sicotte, St-Hyacinthe, Québec, \\ J2S 2M2, Canada
}

\begin{abstract}
Digital dermatitis (DD) is a worldwide infectious disease of cattle that causes lameness, discomfort, and economic losses. The reference standard test to diagnose DD is visual observation in a trimming chute, which cannot be practically performed daily on dairy farms. Moreover, some lesion misclassification may occur using this standard diagnostic method. The possibility of misclassification makes the use of a trimming chute debatable as a perfect reference standard test. The objective of this study was, therefore, to assess the diagnostic accuracy of a commercial borescope and trimming chute exam. The accuracy (sensitivity and specificity) of the tests and DD prevalence were investigated using Bayesian latent class analyses. Our hypothesis was that a commercial borescope can be routinely used to diagnose DD in a milking parlor without previous feet cleaning. A cross-sectional study was performed in a freestall facility. The lesions were scored (M0, M1, M2, M3, M4, M4.1) in the milking parlor with a borescope, followed by an examination in the trimming chute 48 to $72 \mathrm{~h}$ after the borescope exam. A total of 870 hind feet were scored during 2 sessions of trimming chute exams and borescope exams in the milking parlor. The data were analyzed in 2 ways. First, data were dichotomized into DD lesions (M1, M2, M3, M4, M4.1) and absence of DD lesions (M0). Second, data were dichotomized into active DD lesions (M1, M2, M4.1) and inactive lesions (M0, M3, M4). A Bayesian latent class model allowing for conditional dependence between tests was used to estimate tests' accuracy, likelihood ratio, and DD prevalence. When the data were dichotomized into DD lesions (M1-M4.1) versus absence of DD (M0) le-
\end{abstract}

Received June 19, 2019.

Accepted December 16, 2019.

*Corresponding author: s.buczinski@umontreal.ca sions, the sensitivity and specificity of the borescope was 55\% [95\% credible interval (CrI) 40-71\%] and $81 \%$ (95\% CrI $75-88 \%$ ). The sensitivity of trimming chute exams was $79 \%$ (95\% CrI 68-88\%), and specificity was $80 \%$ (95\% CrI 71-89\%). When the data were dichotomized into active lesions (M1, M2, M4.1) versus inactive lesions or absence of lesions (M3, M4, M0), the sensitivity and specificity of the borescope were, respectively, $32 \%$ (95\% CrI 13-58\%) and $91 \%$ (95\% CrI $88-95 \%)$. The sensitivity and specificity of trimming chute exams were $91 \%$ (95\% CrI $81-97 \%$ ) and $81 \%$ (95\% CrI 75-89\%), respectively. In conclusion, it is possible to use the borescope in the milking parlor without cleaning the feet to monitor prevalence of DD lesions. However, an isolated borescope examination, especially for diagnosing active DD lesions, has low sensitivity for use as a surveillance method. For such use, the sensitivity could be improved by repeating the borescope exam on a regular basis.

Key words: dairy cattle, digital dermatitis, diagnostic accuracy, Bayesian latent class model, borescope

\section{INTRODUCTION}

Digital dermatitis (DD) is an infectious disease of cattle with a worldwide distribution (Evans et al., 2016; Anklam et al., 2017). It is the most common foot lesion in confined dairy cattle, affecting 70 to $94 \%$ of herds and approximately $20 \%$ of cows in eastern Canada (Cramer et al., 2018). Digital dermatitis has a great impact on animal welfare due to the high incidence and long duration of the infection (Plummer and Krull, 2017; Solano et al., 2017).

Digital dermatitis causes lameness and discomfort (Gomez et al., 2012). The inflammation caused by DD will affect hoof conformation (Gomez et al., 2015a), with subsequent poor performance in first-lactation heifers (Gomez et al., 2015b). Digital dermatitis is also responsible for economic losses, including milk losses 
(Relun et al., 2013b) and treatment and control expenses (Cha et al., 2010; Relun et al., 2013a). Digital dermatitis is a multifactorial disease, and its exact etiology remains unclear, although bacteria of the genus Treponema seem to play a critical role (Zinicola et al., 2015; Evans et al., 2016; Orsel et al., 2018).

The reference standard test to diagnose DD is direct observation of lesions in the trimming chute (Relun et al., 2011; Solano et al., 2016; Jacobs et al., 2018). However, daily or weekly trimming chute observation to monitor DD prevalence is not practical (Solano et al., 2017). The exam requires labor, as well as a trimming chute, which is not readily available for every dairy herd (Thomsen et al., 2008; Van Hertem et al., 2014; Solano et al., 2017). Nonetheless, detection of DD lesions and their early treatment are fundamental to control DD (Döpfer et al., 2012; Stokes et al., 2012). Recently, the use of visual inspection in a trimming chute as a "gold standard" has been questioned. Diagnostic tests using visual inspection depend on the skills and experience of the operators and have several disadvantages such as extra labor and facilities requirements (DuttonRegester et al., 2018). Washing versus not washing the feet could also affect test accuracy (Yang and Laven, 2019). Therefore, this exam is prone to being subjective and can be imperfect; consequently, it has been suggested to use a latent class model approach to estimate its accuracy for bovine lameness (Dutton-Regester et al., 2018). In fact, latent class models have already been used to estimate DD prevalence and accuracy of inspection in milking parlor to diagnose DD (Yang et al., 2017).

Therefore, alternative methods to trimming chute observation have been investigated for easier diagnosis during daily herd operations; for instance, during milking time (Thomsen et al., 2008; Relun et al., 2011; Solano et al., 2017). Among these methods, a borescope was used to visualize the plantar part of the hind feet. Similar to a medical endoscope, a commercial borescope is an optical device consisting of an illuminated camera or objective lens linked to a display by an optical system surrounded by optic fibers and a rigid or flexible tube. It can be wirelessly connected to an imaging device (e.g., a tablet or cell phone). Borescopes are used in different fields where a visual inspection in an inaccessible area is needed (e.g., mechanical engines, hydraulics) and have been used to diagnose DD (Laven, 1999; Stokes et al., 2012). However, the use of this method has been limited by its costs and its effect on milking time duration (Thomsen et al., 2008; Relun et al., 2011; Solano et al., 2017; Cramer et al., 2018). Currently, several affordable Wi-Fi-compatible models of flexible borescope are available on the market. Their flexibility could help better visualize DD lesions. For these reasons, it would be meaningful to determine the accuracy of this type of commercial borescope for DD diagnosis in dairy cattle.

Our hypothesis was that a commercial borescope could be used as diagnostic tool in a milking parlor on unwashed feet to detect active DD lesions and monitor DD prevalence.

The objective of this study was to assess the accuracy, defined as sensitivity ( $\mathbf{S e})$, specificity $(\mathbf{S p})$, and derived likelihood ratios ( $\mathbf{L R})$, of a commercial borescope for the diagnosis of DD lesions in the milking parlor versus that of direct observation in a trimming chute using a latent class analysis.

\section{MATERIALS AND METHODS}

The study was completed in accordance with the guidelines of the Institutional Animal Care Committee (CÉUA) of the Université de Montréal (CÉUA protocol: \#16-Rech-1826).

\section{Definitions}

In this article, sensitivity is defined as the proportion of diseased animals that test positive; specificity is the proportion of nondiseased animals that test negative (Dohoo et al., 2009). A likelihood ratio is defined as the percentage of diseased individuals with a given test result, divided by the percentage of nondiseased individuals with the same test result. A positive likelihood ratio $(\mathbf{L R}+)$ is the percentage of diseased individuals with a positive test result (Se), divided by the percentage of nondiseased individuals with a positive test result $(1-\mathrm{Sp})$. A negative likelihood ratio $(\mathbf{L R}-)$ is the percentage of diseased individuals with a negative test result $(1-\mathrm{Se})$ divided by the percentage of nondiseased individuals with a negative test result $(\mathrm{Sp})$ (Timsit et al., 2018).

\section{Study Design}

Data used to assess the accuracy of a commercial borescope were obtained from a nonrandomized clinical trial evaluating a new experimental protocol for $\mathrm{DD}$ control in a single freestall dairy herd (unpublished data). The sample size was calculated with an expected prevalence of DD of $20 \%$. Thus, with 1,000 observations, we anticipated obtaining 200 affected and 800 healthy feet. We considered values of 50 and $80 \%$ as probable values for the $\mathrm{Se}$ and $\mathrm{Sp}$ of the borescope, respectively. With these values, we estimated that Se of the borescope could be estimated with a precision of 
\pm 7 percentage points, whereas Sp could be estimated with precision of \pm 3 percentage points. These calculations, however, did not account for imprecision of the trimming chute exam and are, thus, approximate and an overestimation of the level of precision that could be achieved. The trial was conducted between April 21 and July 25, 2017.

\section{Source Population, Target Populations, and Study Sample}

The source population was a commercial, 250-milking-cow freestall dairy, which was part of the clients of the Bovine Ambulatory Clinic of the Faculté de Médecine Vétérinaire of the Université de Montréal.

Cows were milked 3 times per day $(0415,1215$, and $2015 \mathrm{~h}$ ) in a rotary milking parlor (28 places). The average $305-\mathrm{d}$ milk production was $11,800 \mathrm{~kg}$. The floor was slatted concrete. The bedding area was a mixture of wheat straw and limestone, which was cleaned daily and changed twice a week. An alley freestall cleaner passed 4 times per day.

The farm's DD control program included the use of a footbath $2 \mathrm{~d} /$ wk using a $5 \%$ copper sulfate solution. The farm-trimming schedule was 3 times per year for cows and twice a year for heifers.

Milking cows present in the herd during the clinical trial were considered sample units. The unit of analysis was the plantar surface of the hind feet. More precisely, in our study, each single plantar face of the hind feet was considered a unit of analysis. Typically, $94 \%$ of DD lesions are present on the hind limbs and $85 \%$ are localized on the skin between the heel bulbs (Cramer et al., 2008; Relun et al., 2011; Solano et al., 2017).

A commercial borescope (WF200 WiFi Endoscope, Teslong Technology Ltd., Shenzhen, Guangdong, China) connected by Wi-Fi to an iPad (Apple Inc., Cupertino, CA) was used to assess DD lesions. Lighting was provided by the LED lamps incorporated in the borescope.

\section{Classification of Lesions}

Lesions were classified using the categories described by Döpfer et al. (1997) and modified by Berry et al. (2012). Briefly, DD lesions were classified into 6 categories: M0 (skin without a sign of DD), M1 (ulcerative reddish lesion of $\mathrm{DD}<2 \mathrm{~cm}$ in diameter), M2 (ulcerative red-to-gray lesion $>2 \mathrm{~cm}$ in diameter), M3 (healing tetracycline-treated lesions, with a blackish scab and with a trace of tetracycline), M4 (chronic proliferative gray lesion), and M4.1 (chronic proliferative lesion with an ulcerative reddish lesion).

\section{Assessment of Lesions in the Milking Parlor}

Evaluation of lesions in the milking parlor was performed by 2 evaluators (SF and $\mathrm{AD}$ ). Feet were not washed before evaluation. Scoring was done at midday during the second daily milking, 48 to $72 \mathrm{~h}$ before the scheduled hoof trimming session in the chute. We aimed to score all milking cows available in the herd at that time. A second scoring session was conducted a few months later immediately before the subsequent scheduled trimming session. Images of the plantar aspect of the hind limb were displayed on the tablet (iPad, Apple Inc.) as video. Lesions of the plantar aspect of the hind limb were scored by the 2 assessors and a consensus score was reached. Scores were communicated to another person, who recorded the data.

\section{Chute Assessment}

Hoof trimming was performed by a professional trimmer using a hydraulic standing trimming chute. Evaluation of DD lesions in the chute was made by one trained observer (SF) positioned approximatively $50 \mathrm{~cm}$ behind the trimmer. The feet were not cleaned before evaluation. The diagnosis was made before trimming and without any particular manipulations of the digits. No treatment was applied between milking parlor and chute assessment.

All collected scores were recorded on a paper form and transferred to an Access database afterward (Microsoft Corp., Redmond, WA).

\section{Evaluation of Interobserver Agreement for Borescope Exam}

Before the study, the first author was trained to identify DD lesions. This training consisted of 2 classroom sessions with a PowerPoint (Microsoft Corp.) presentation using digital color photographs to demonstrate each DD stage. Each PowerPoint presentation contained 40 pictures of feet. After presentation, each photo was scored by the first author. The misclassified pictures were discussed with the senior author to standardize future scoring.

Interobserver agreement for borescope examination was determined using scores obtained from a subgroup of cows (the cows between 0 DIM and around 120 DIM) that were evaluated in parallel and independently by the 2 evaluators. Agreement beyond chance was investigated using Cohen's kappa ( $\boldsymbol{\kappa})$. To compute $\kappa$, lesions were dichotomized using 2 classification methods: (1) DD lesions (M1, M2, M3, M4, M4.1) and absence of DD lesions (M0); and (2) active DD lesions (M1, M2, M4.1) 
and inactive DD lesions/absence of DD lesions (M3, $\mathrm{M} 4, \mathrm{M} 0)$. The $\kappa$ results were interpreted using the $\kappa$ mean estimate following previously reported guidelines (Dohoo et al., 2009): $\leq 0=$ poor agreement, 0.01 to 0.2 $=$ slight agreement, 0.21 to $0.4=$ fair agreement, 0.41 to $0.6=$ moderate agreement, 0.61 to $0.8=$ substantial agreement, 0.81 to $1=$ almost perfect agreement .

\section{Agreement Between Borescope and Trimming Chute Exams}

We used all observations where both a borescope and trimming chute measurement were available for assessing agreement between borescope and trimming exam. Agreement between scores obtained using the borescope and in the trimming chute was investigated using $\kappa$ measures (Dohoo et al., 2009). Agreement was calculated for the 2 lesion classifications previously described (any DD lesions vs. no DD lesions; active DD lesions vs. no active DD lesions).

The magnitude of the discrepancy between borescope and trimming chute exams was computed using weighted kappa ( $\boldsymbol{\kappa}_{\mathrm{w}}$; Cohen, 1968; Dohoo et al., 2009). Lesions were assumed to be ordered as follows: M2 > M4.1 > M1 > M4 > M3 > M0 (Solano et al., 2017). The results were interpreted as described above.

\section{Accuracy of Borescope and Trimming Chute Exams}

Results from the borescope and trimming chute exams were cross-tabulated using the 2 proposed categorizations (any DD lesions vs. no DD lesions; active DD lesions vs. no active DD lesions), thus creating one $2 \times 2$ table for each of these 2 categorizations. Accuracy parameters as well as the true prevalence of lesions were then estimated using a latent class model for comparing 2 conditionally independent tests in a single population. Our target population was the feet of milking Holstein dairy cows. The first latent variable in the first categorization scheme was the presence of DD lesions indicating that the feet were previously or currently exposed to this infectious disease. The second latent variable in the second categorization was a foot that was under active infection. This second latent variable classification would represent the feet that are considered a major concern for animal welfare because they cause important pain in affected cows.

In most latent class models, the number of sampled animals in the population is modeled using a binomial distribution, thus assuming that the sample size is infinite. For the current study, however, most of the cows available in the studied herd were evaluated, so this herd is clearly a finite population. Therefore, we used the Bayesian superpopulation approach described (Jones and Johnson, 2016) and appropriate for finite populations. We also considered in this model conditional dependence between tests, because both tests rely on visual (direct or indirect) inspection. Thus, tests could be positively correlated. To achieve that, 2 parameters describing covariances between tests, conditional on the true disease status, were added to the model: covariance sensitivity (covSe) and covariance specificity (covSp). Using this approach, the prior distributions for covSe and covSp can be described as uniform distribution (Unif) with specific bounds:

$$
\begin{aligned}
& \operatorname{covSe} \sim \operatorname{Unif}[0, \min (\mathrm{Se} 1, \mathrm{Se} 2)-\mathrm{Se} 1 \times \mathrm{Se} 2] \\
& \operatorname{covSp} \sim \operatorname{Unif}[0, \min (\mathrm{Sp} 1, \mathrm{Sp} 2)-\mathrm{Sp} 1 \times \mathrm{Sp} 2] .
\end{aligned}
$$

Prior information was elicited from 3 of the co-authors before data analysis: SF (a third-year resident student, candidate of the European College of Bovine Health Management), $\mathrm{AD}$, and $\mathrm{MR}$ (both diplomates of the American College of Veterinary Surgeons with expertise in bovine lameness). These experts independently provided the most probable prevalence of DD lesions (in general) and of active DD lesions in freestalls in Québec, along with 5th or 95th percentile, representing the uncertainty around that estimate. The same process was used to elicit borescope and trimming chute Se and Sp.

This information was combined using the median values of the best guesses and minimum and maximum values indicated by the experts as 5th and 95th percentiles, respectively (Branscum et al., 2005). The best guess for the prevalence of any DD lesions in freestalls in Québec was 25\% (10-70\%). The best guess for the Se of the borescope for estimating DD lesions was $55 \%$ (40-70\%), and for Sp, 80\% (70-99\%). For the trimming chute, the best guess for Se was $80 \%$ (70-99\%), and for Sp, $80 \%$ (60-99\%). For active DD lesions, the best guess for the prevalence was $10 \%(5-35 \%)$. The best guess for Se of the borescope for estimating these was $40 \%(20-60 \%)$, and for Sp, $85 \%$ (75-99\%). For the trimming chute, the best guess for Se for diagnosing active DD lesions was $90 \%$ (80-99\%), and for Sp, $90 \%$ (80-99\%).

These values were then used to determine the corresponding $\beta$ distributions using the best guess as mode and minimum or maximum as 5th or 95th percentile, respectively. To investigate how sensitive the model was to the chosen priors, the model was also run with a set of priors using the elicited priors for trimming chute 
Table 1. Number $(\%)$ of digital dermatitis lesions identified using trimming chute and borescope in the milking parlor exams in 1 herd in Québec, Canada

\begin{tabular}{|c|c|c|c|c|c|c|c|c|}
\hline Exam & \multicolumn{7}{|c|}{ Classification $^{1}$} & Total \\
\hline Trimming chute & $\begin{array}{l}733 \\
(69.0 \%)\end{array}$ & $\begin{array}{l}147 \\
(14.0 \%)\end{array}$ & $\begin{array}{l}91 \\
(8.5 \%)\end{array}$ & $\begin{array}{l}1 \\
(0.1 \%)\end{array}$ & $\begin{array}{l}51 \\
(4.8 \%)\end{array}$ & $\begin{array}{l}39 \\
(3.6 \%)\end{array}$ & $\begin{array}{l}2 \\
(0.2 \%)\end{array}$ & $\begin{array}{c}1,064 \\
(100 \%)\end{array}$ \\
\hline Borescope & $\begin{array}{l}647 \\
(71.5 \%)\end{array}$ & $\begin{array}{l}77 \\
(8.5 \%)\end{array}$ & $\begin{array}{l}15 \\
(1.7 \%)\end{array}$ & $\begin{array}{l}15 \\
(1.7 \%)\end{array}$ & $\begin{array}{l}124 \\
(13.8 \%)\end{array}$ & $\begin{array}{c}8 \\
(0.8 \%)\end{array}$ & $\begin{array}{l}18 \\
(2.0 \%)\end{array}$ & $\begin{array}{c}904 \\
(100 \%)\end{array}$ \\
\hline
\end{tabular}

${ }^{1}$ Digital dermatitis (DD) lesions were classified in 6 categories according to Döpfer et al. (1997) and Berry et al. (2012): M0 (skin without sign of DD), M1 (ulcerative reddish lesion of DD less than $2 \mathrm{~cm}$ in diameter), M2 (ulcerative red-to-gray lesion more than $2 \mathrm{~cm}$ in diameter), M3 (healing oxytetracycline treated lesions, with a blackish scab and with trace of oxytetracycline), M4 (chronic proliferative gray lesion), M4.1 (chronic proliferative lesion with an ulcerative reddish lesion), and NC (nonclassifiable).

accuracy parameters and for the prevalence but vague priors of the form $\beta(1.0,1.0)$ for borescope accuracy parameters.

Models were run using OpenBUGS (version 3.2.3 rev 2012, Medical Research Council, UK). Three chains were run, and a burn-in period of 5,000 iterations was used for all analyses. History, autocorrelation, and Brooks-Gelman-Rubin plots were investigated to assess chain convergence. Thinning was used when important autocorrelation was noted. Posterior distributions were described using Markov chain Monte Carlo approximations containing 60,000 values (after thinning or discarding of inappropriate Markov chains). The fit of the model was determined using the deviance information criterion (DIC; Quintero and Lesaffre, 2018). The OpenBUGS model and data are provided in Supplemental File S1 (https://doi.org/10.3168/jds .2019-17129).

\section{RESULTS}

Two sessions of hoof trimming and borescope exams were performed in the herd during the study period. A total of 1,064 hind feet were evaluated in the trimming chute. Scoring distribution of the lesions was as follows: 733 M0 (69\%), 147 M1 (14\%), 91 M2 (8.5\%), $1 \mathrm{M} 3$ (0.10\%), $51 \mathrm{M} 4$ (4.8\%), and $39 \mathrm{M} 4.1$ (3.6\%); 2 $(0.2 \%)$ were considered unclassifiable (Table 1$)$. A total of 904 hind feet were evaluated with the borescope in the milking parlor. The scoring of the lesions was 647 M0 (71.5\%), 77 M1 (8.5\%), 15 M2 (1.65\%), 15 M3 (1.65\%), $124 \mathrm{M} 4$ (13.8\%), and 8 M4.1 (0.8\%); $18(2 \%)$ were considered unclassifiable (i.e., when a foot was in a dung pat, or because of the foot's conformation, or when a bandage was present; Table 1). A total of 870 foot measurements with both trimming chute and borescope exams could be used for the analyses. The cross-tabulated results of exams in the milking parlor using the borescope and during chute trimming for diagnosing DD lesions per the categories DD lesions
(M1 to M4.1) versus no DD lesions (M0) and active DD lesions (M1, M2, M4.1) are presented in Tables 2, 3 , and 4 , respectively.

\section{Interobserver Agreement for the Borescope Exam}

One hundred seventy-six cows were examined independently by the 2 observers during the borescope exams. The percent of overall agreement was $95.5 \%$ for detecting DD lesions versus absence of DD lesions. The mean $(95 \%$ CI $) \kappa$ value was $0.87(0.79-0.96)$, indicative of substantial to almost perfect agreement. No active DD lesions were observed; thus, interobserver agreement for active versus inactive or absence of lesions could not be computed.

\section{Agreement Between Borescope and Trimming Chute Exams for Grading DD Lesions}

The $\kappa$ value between the borescope and the trimming chute for detecting any DD lesions versus no DD lesions was 0.57 (95\% CI: 0.51-0.63). The $\kappa$ value was

Table 2. Cross-tabulated results of borescope and trimming chute exams conducted on 870 feet for diagnosing different type of digital dermatitis (DD) lesions in 1 dairy herd in Québec, Canada ${ }^{1}$

\begin{tabular}{lrrrrcrr}
\hline & \multicolumn{7}{c}{ Borescope } \\
\cline { 2 - 8 } $\begin{array}{l}\text { Trimming } \\
\text { chute }\end{array}$ & M0 & M3 & M4 & M1 & M4.1 & M2 & Total \\
\hline M0 & 530 & 1 & 48 & 3 & 0 & 0 & 582 \\
M3 & 1 & 0 & 0 & 0 & 0 & 0 & 1 \\
M4 & 14 & 0 & 31 & 0 & 0 & 0 & 45 \\
M1 & 68 & 3 & 21 & 33 & 2 & 1 & 128 \\
M4.1 & 4 & 3 & 17 & 9 & 2 & 1 & 36 \\
M2 & 18 & 7 & 7 & 30 & 3 & 13 & 78 \\
Total & 635 & 14 & 124 & 75 & 7 & 15 & 870 \\
\hline
\end{tabular}

${ }^{1}$ Score definition: M0 (skin without sign of DD), M1 (ulcerative reddish lesion of $\mathrm{DD}<2 \mathrm{~cm}$ in diameter), M2 (ulcerative red-to-gray lesion $>2 \mathrm{~cm}$ in diameter), M3 (healing oxytetracycline treated lesions, with a blackish scab and with trace of oxytetracycline), M4 (chronic proliferative gray lesion), and M4.1 (chronic proliferative lesion with an ulcerative reddish lesion). 
Table 3. Cross-tabulated results of borescope and trimming chute exams conducted on 870 feet for diagnosing any digital dermatitis (DD) lesion [(M1, M2, M3, M4, or M4.1) vs. M0] in 1 dairy herd in Québec, Canada ${ }^{1}$

\begin{tabular}{lccc}
\hline Result & Borescope + & Borescope - & Total \\
\hline Trimming chute + & 183 & 105 & 288 \\
Trimming chute - & 52 & 530 & 582 \\
Total & 235 & 635 & 870 \\
\hline
\end{tabular}

${ }^{1} \mathrm{~A}$ positive test was defined as the presence of any M1, M2, M3, M4, or M4.1 lesion. A negative test was any M0 score. Score definition: M0 (skin without sign of DD), M1 (ulcerative reddish lesion of DD $<2 \mathrm{~cm}$ in diameter), M2 (ulcerative red-to-gray lesion $>2 \mathrm{~cm}$ in diameter), M3 (healing oxytetracycline treated lesions, with a blackish scab and with trace of oxytetracycline), M4 (chronic proliferative gray lesion), and M4.1 (chronic proliferative lesion with an ulcerative reddish lesion).

0.47 (95\% CI: $0.40-0.54)$ for DD active lesions versus inactive or absent DD lesions (moderate to substantial agreement). When comparing borescope and trimming chute exam agreement over the full range of lesion categories, a $\kappa_{\mathrm{w}}$ value of 0.48 (95\% CI: $\left.0.43-0.53\right)$ was obtained, indicating moderate agreement.

\section{Accuracy of Borescope and Trimming Chute Exams for Diagnosing DD Lesions in General}

Posterior distributions for Se, Sp, LR+, LR-, covSe, covSp, and DD lesion prevalence (in general) are reported in Table 5. Using the elicited informative priors on all unknown parameters, borescope Se and Sp were
Table 4. Cross-tabulated results of borescope and trimming chute exams conducted on 870 feet for diagnosing active digital dermatitis (DD) lesions [(M1, M2, or M4.1) vs. (M0, M3 or M4)] in 1 dairy herd in Québec, Canada ${ }^{1}$

\begin{tabular}{lccc}
\hline Result & Borescope + & Borescope - & Total \\
\hline Trimming chute + & 94 & 148 & 242 \\
Trimming chute - & 3 & 625 & 628 \\
Total & 97 & 773 & 870 \\
\hline
\end{tabular}

${ }^{1} \mathrm{~A}$ positive test was defined as the presence of any M1, M2, or M4.1 lesion. A negative test was any M0, M3, or M4 score. Score definition: M0 (skin without sign of DD), M1 (ulcerative reddish lesion of $\mathrm{DD}<2 \mathrm{~cm}$ in diameter), M2 (ulcerative red-to-gray lesion $>2 \mathrm{~cm}$ in diameter), M3 (healing oxytetracycline treated lesions, with a blackish scab and with trace of oxytetracycline), M4 (chronic proliferative gray lesion), and M4.1 (chronic proliferative lesion with an ulcerative reddish lesion).

estimated at 55\% (95\% CrI: $39-71 \%)$ and $81 \%(95 \%$ CrI: $75-88 \%$ ), respectively. Trimming chute Se and Sp were estimated at $79 \%(95 \%$ CrI: $68-88 \%)$ and $80 \%$ (95\% CrI: 71-89\%). Borescope exam LR+ was estimated at 2.9 (95\% CI: 1.7-5.2) and LR- was 0.55 (95\% CI: $0.34-0.79)$. Using vague priors on borescope accuracy parameters resulted in slightly higher borescope Se and Sp (72 and 87\%, respectively; see Table 5).

\section{Accuracy of Borescope and Trimming Chute Exams for Diagnosing Active $D D$ Lesions}

Posterior distributions for Se, Sp, LR+, LR-, covSe, covSp, and active DD lesion prevalence are reported in

Table 5. Results from the Bayesian latent class models estimating using borescope and trimming chute exam for diagnosing any digital dermatitis (DD) lesion (M1, M2, M3, M2, or M4.1) in 1 dairy herd in Québec, Canada

\begin{tabular}{|c|c|c|c|c|}
\hline Variable $^{1}$ & \multicolumn{2}{|c|}{ Informative $\beta$ priors on all parameters } & \multicolumn{2}{|c|}{ Vague priors on borescope accuracy parameters ${ }^{2}$} \\
\hline \multicolumn{5}{|l|}{ Trimming } \\
\hline $\mathrm{Sp}$ & Beta $(14.8,4.5)$ & $80(71,89)$ & Beta $(14.8,4.5)$ & $81(71,91)$ \\
\hline $\mathrm{LR}+$ & - & $3.9(2.6,7.6)$ & - & $4.2(2.6,8.9)$ \\
\hline LR- & - & $0.26(0.15,0.41)$ & - & $0.26(0.15,0.41)$ \\
\hline Sp & Beta $(48.3,12.8)$ & $81(75,88)$ & Beta $(1.0,1.0)$ & $87(72,99)$ \\
\hline $\mathrm{LR}+$ & - & $2.9(1.7,5.2)$ & - & $5.8(0.7-91.6)$ \\
\hline $\mathrm{LR}-$ & - & $0.55(0.34,0.79)$ & - & $0.31(0.01,1.12)$ \\
\hline Prevalence among sampled & Beta $(3.1,7.4)$ & $23(10,34)$ & Beta $(3.1,7.4)$ & $24(9,36)$ \\
\hline Population prevalence & - & $22(9,34)$ & - & $24(9,35)$ \\
\hline Cov Se & Unif $(a, b)^{4}$ & $0.02(-0.09,0.13)$ & Unif $(a, b)^{4}$ & $0.02(-0.09,0.13)$ \\
\hline Cov Sp & $\operatorname{Unif}(\mathrm{c}, \mathrm{d})^{4}$ & $0.10(0.04,0.15)$ & Unif $(\mathrm{c}, \mathrm{d})^{4}$ & $0.06(0.00,0.16)$ \\
\hline
\end{tabular}

${ }^{1}$ Sensitivity (Se), specificity (Sp), covariance between sensitivity of borescope and trimming chute exam among cows with an active DD lesion (Cov Se), covariance between specificity of borescope and trimming chute exam among cows without an active DD lesion (Cov Sp), likelihood ratio positive $(\mathrm{LR}+)$, likelihood ratio negative ( $\mathrm{LR}-$ ), deviance information criterion (DIC), uniform distribution (Unif).

${ }^{2}$ Thinning of 5 was applied to improve autocorrelation and each chain was run for 100,000 iterations (generating 20,000 samples per chain). ${ }^{3}$ Median estimate (\%) and $95 \%$ credible intervals are reported to describe the posterior distribution.

${ }^{4}$ Where a is $(1-$ trimming Se $) \times($ Se boroscopy -1$)$; b is the smallest Se between trimming chute and borescope minus the product of these $\mathrm{Se}$; $\mathrm{c}$ is (trimming $\mathrm{Sp}-1) \times(1-$ boroscopy $\mathrm{Sp})$; and $\mathrm{d}$ is the smallest Sp between trimming and borescope minus the product of these Sp. 
Table 6. When using the elicited priors, the Se of the borescope was estimated at 32\% (95\% CrI: 13-58\%) and Sp was 91\% (95\% CrI: 88-95\%). The Se of the trimming chute was 91\% (95\% CrI: $82-97 \%$ ) and Sp was $81 \%$ (95\% CrI: $75-89 \%$ ).

\section{DISCUSSION}

In this study, we found that the specificity of the commercial borescope was adequate for a rapid screening of digital infection on uncleaned feet in the milking parlor. However, it lacked sensitivity to diagnose active DD lesions or to detect DD lesions if used on a single occasion.

We considered the visual inspection in the trimming chute as an "imperfect test" using a latent class model approach, as recently suggested (Dutton-Regester et al., 2018). This was especially important because the feet were not washed in the trimming chute.

In our study, we used a model that allowed for conditional dependence between 2 tests. Conditional dependence could arise when 2 tests share a similar biological basis (Branscum et al., 2005). Both tests (borescope and trimming chute) were based on visual observation of DD lesions and thus shared the same biological basis.

The trimming chute examination was not perfectly accurate as anticipated. In other studies, visual inspec- tion in the chute was considered the gold standard test (Relun et al., 2011; Solano et al., 2017; Cramer et al., 2018). In these studies, the feet were cleaned before the visual assessment using paper towels (Solano et al., 2017; Cramer et al., 2018) or using brush and water (Relun et al., 2011). In our study, no specific feet cleaning protocol was made before the assessment. It is therefore important to consider that the visual observation made in our study differed slightly from chute examinations reported in previous studies. This is another reason for not considering this chute examination as a true gold standard test but rather as an imperfect reference standard test that can also misclassify some health statuses. Nevertheless, even with a feet washing protocol in place, we can hardly consider a trimming chute exam as a gold standard test for diagnosing DD lesions or active DD lesions, because evaluation and classification of these lesions is difficult, even for experts. Moreover, in the milking parlor, not washing or cleaning the feet could increase misclassified feet due to difficulties of observing some lesions, as shown in a previous study comparing visual feet assessment pre- and post-washing in the milking parlor (Yang and Laven, 2019). In that study, sensitivity almost doubled when comparing before and after feet washing with an almost $94 \%$ chance that post-washing sensitivity was greater than pre-washing. No specific information is available

Table 6. Results from the Bayesian latent class models estimating exam with borescope and trimming chute exam accuracy for diagnosing active digital dermatitis (DD) lesions (M1, M2, or M4.1) in 1 dairy herd in Québec, Canada (each model was run to obtain a Monte Carlo simulation containing 60,000 values after thinning)

\begin{tabular}{|c|c|c|c|c|}
\hline Variable $^{1}$ & \multicolumn{2}{|c|}{ Informative $\beta$ priors on all parameters ${ }^{2}$} & \multicolumn{2}{|c|}{ Vague priors on borescope accuracy parameters ${ }^{3}$} \\
\hline $\mathrm{Se}$ & Beta $(42.6,5.6)$ & $91(81,97)$ & Beta $(42.6,5.6)$ & $91(82,97)$ \\
\hline Sp & Beta $(42.6,5.6)$ & $81(75,89)$ & Beta $(42.6,5.6)$ & $83(77,91)$ \\
\hline $\mathrm{LR}+$ & - & $4.9(3.5,8.7)$ & - & $\mathrm{DNC}$ \\
\hline LR- & - & $0.11(0.04,0.23)$ & - & $0.10(0.04,0.22)$ \\
\hline Sp & Beta $(46.3,9.0)$ & $91(88,95)$ & Beta $(1.0,1.0)$ & $97(88,100)$ \\
\hline $\mathrm{LR}+$ & - & $3.6(1.2,9.4)$ & - & $\mathrm{DNC}$ \\
\hline LR- & - & $0.75(0.45,0.98)$ & - & $0.42(0.07,1.0)$ \\
\hline Prevalence among sampled & Beta $(5.2,38.8)$ & $12(5,21)$ & $\operatorname{Beta}(5.2,38.8)$ & $14(7,23)$ \\
\hline Population prevalence & - & $12(5,21)$ & - & $14(7,22)$ \\
\hline Cov Se & Unif $(a, b)^{5}$ & $0.01(-0.04,0.06)$ & $\operatorname{Unif}(\mathrm{a}, \mathrm{b})^{5}$ & $0.03(-0.02,0.10)$ \\
\hline Cov Sp & Unif $(\mathrm{c}, \mathrm{d})^{5}$ & $0.07(0.04,0.09)$ & Unif $(\mathrm{c}, \mathrm{d})^{5}$ & $0.02(0.00,0.09)$ \\
\hline
\end{tabular}

${ }^{1}$ Sensitivity (Se), specificity (Sp), covariance between sensitivity of borescope and trimming chute exam among cows with an active DD lesion (Cov Se), covariance between specificity of borescope and trimming chute exam among cows without an active DD lesion (Cov Sp), likelihood ratio positive $(\mathrm{LR}+)$, likelihood ratio negative (LR-), deviance information criterion (DIC), uniform distribution (Unif), did not converge (DNC). ${ }^{2}$ Thinning of 5 was applied to improve autocorrelation and each chain was run for 100,000 iterations (generating 20,000 samples per chain). ${ }^{3}$ Thinning of 10 was applied to improve autocorrelation and each chain was run for 200,000 iterations (generating 20,000 samples per chain). ${ }^{4}$ Median estimate (\%) and $95 \%$ credible intervals are reported to describe the posterior distribution.

${ }^{5}$ Where a is $(1-$ trimming Se $) \times($ Se boroscopy -1$)$; b is the smallest Se between trimming chute and borescope minus the product of these $\mathrm{Se}$; is (trimming $\mathrm{Sp}-1) \times(1-$ boroscopy Sp); and $\mathrm{d}$ is the smallest Sp between trimming and borescope minus the product of these Sp. 


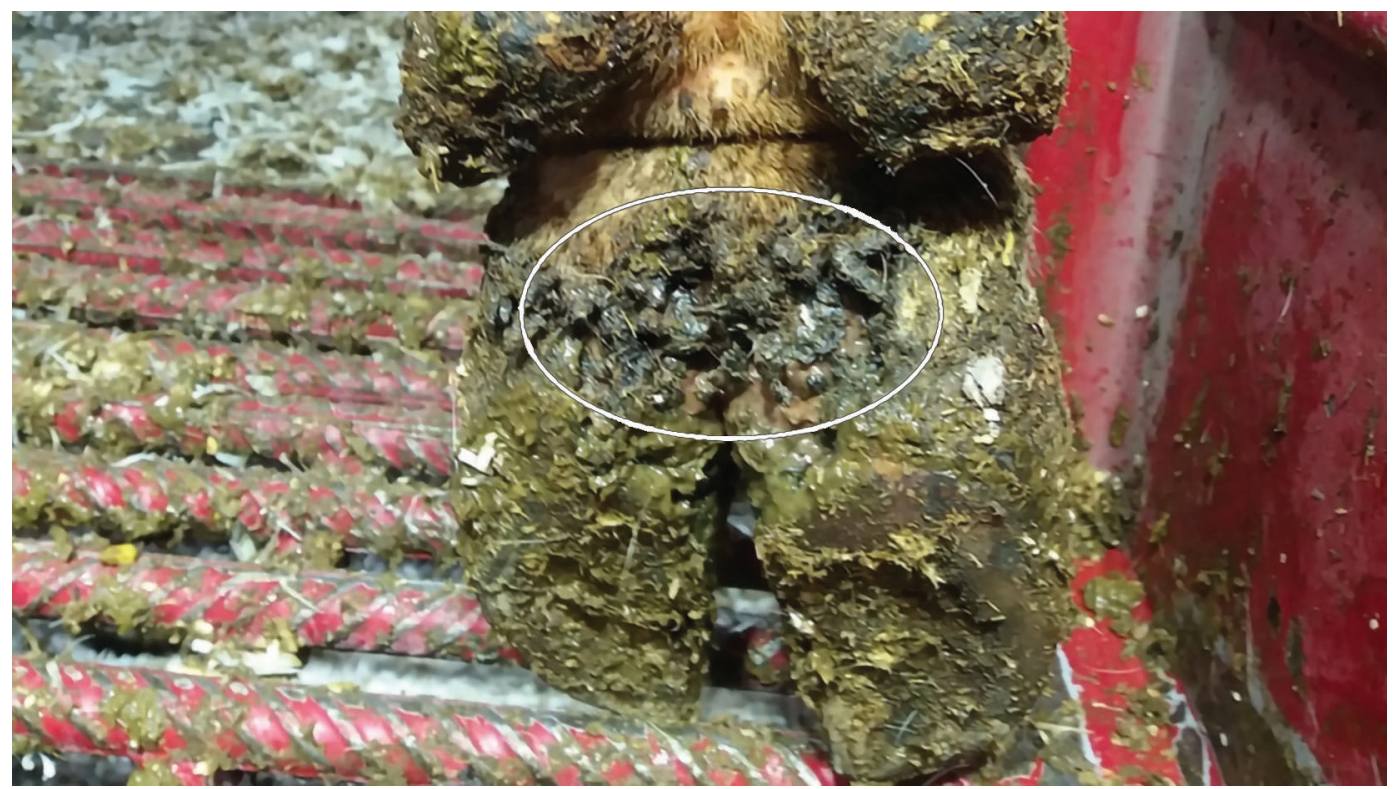

Figure 1. Examination in a standing trimming chute of an uncleaned foot. The plantar crust of manure can be confounded with an M4 lesion.

on the impact of washing feet in the trimming chute on visual assessment accuracy.

In the present study, both tests yielded similar specificity (around 80-90\%); consequently, around 10 to $20 \%$ false positive fraction. Other studies reported the possibility of overestimating M4 prevalence when a diagnostic tool is used to score DD in the milking parlor (Relun et al., 2011; Stokes et al., 2012). Our hypothesis is that false positives may be due to misclassification of M4 lesions. Our experience suggests that dirt can be confounded with a chronic proliferative lesion (see Figure 1). This is most likely the case in the current study, because the feet were not cleaned before the exams. It has been reported that scoring DD in the milking parlor without washing the hind feet greatly reduces detection of DD lesions (Cramer et al., 2018; Yang and Laven, 2019) and can lead to an underestimation of the true prevalence of DD (Yang and Laven, 2019). The dirtiness of the feet could certainly explain, in part, the lower Se of the commercial borescope. We elected not to clean the feet in the milking parlor before examination based on anecdotal reports of potential bacterial contamination during milking (Cramer et al., 2018).

In all models, the sensitivity of the borescope was lower than that of the trimming chute exam, which was expected and is most likely due to M1 lesions being missed during borescopy. Indeed, other studies have reported the difficulty of diagnosing M1 lesions, and they tend to be misclassified (Relun et al., 2011; Stokes et al., 2012; Jacobs et al., 2017; Solano et al., 2017;
Cramer et al., 2018). In our study, M1 was the most prevalent lesion identified during chute trimming exams (14\%). The distribution of lesions may have affected the accuracy of the borescope in our study because of so-called spectrum bias. Spectrum bias occurs when the accuracy measures of a test are influenced by the case mix of patients in the study sample (in our case, the distribution of cows with various DD lesions) and the prevalence and expression of the disease. In many diseases, as previously mentioned, the distinction between an absent and present condition is an "underlying continuum between 'barely present' to 'clearly present,' which can be affected by the prevalence of the target condition" (Leeflang et al., 2009, 2013). In this case, the shape of the distribution of this continuum may depend on the prevalence. In contrast, the false positive cases may depend on the prevalence of other non-DD feet lesions. The definition of M1 lesions is somewhat unclear because it includes all ulcerative lesions with a diameter $<2 \mathrm{~cm}$. Very small lesions that are difficult to detect by direct observation are likely to go unnoticed when using the borescope or could be misdiagnosed as interdigital dermatitis (Manske et al., 2002).

Another possible explanation for the low sensitivity of the borescope is the difference in the angulation of the feet when observed in the milking parlor versus in the trimming chute. The latter allows for a complete visualization of the feet's surface, whereas when observing feet in the milking parlor with the borescope, visualization of this area is impaired by feet's conformation 
(e.g., heel height), presence of feces, and position of the cow in the milking parlor (Thomsen et al., 2008; Yang et al., 2017).

The quality of the borescope itself and the resolution of the image on both the tablet (iPad) and the borescope (number of pixels) can also explain the lower sensitivity. Different borescope models with different prices $(\$ 10-\$ 1,000)$ are available on the market. In our study, we chose a medium-low-price model (Can $\$ 110$; approximately US\$84) with the extremity at an angle of $0^{\circ}$. Some expensive rigid borescopes have a direction of view of up to $140^{\circ}$, which can improve the observation of more interdigital lesions. The tablet model may affect test accuracy, because the image's resolution varies between models $(2,048 \times 1,536$ resolution at 8 megapixels per inch for the iPad Air vs. 3,264 × 2,448 pixels at 9 megapixels for the more recent iPad Pro).

The accuracy of the borescope in the present study was different from that reported in other studies using borescopes in the milking parlor (Laven, 1999; Stokes et al., 2012). It is difficult to directly compare these results because the previous authors used a different type of borescope (a rigid borescope specially designed for study needs). In the earlier studies, enrollment of cases was different and a very different classification of the lesions was used. Furthermore, the feet were washed before assessment in the earlier studies.

The results of the borescope's accuracy to diagnose DD in milking parlor, as found in our study, are comparable with direct observations in the milking parlor reported by Cramer et al. (2018), who reported a sensitivity of $58 \%$ and a specificity of $95.3 \%$. In that study, the feet were not washed before assessment and the authors used the same classification that we used in the current study.

Despite the previous limitations, using a borescope in a milking parlor to diagnose DD still has some advantages. Borescopes are affordable, and they can be used regularly and easily compared with trimming chute exams to identify cows with DD lesions. Therefore, they could improve the treatment and control strategy as well as providing prevalence monitoring between trimming sessions. We believe that using a borescope on a regular basis (e.g., once a week) could lead to an overall higher sensitivity compared with a trimming chute exam 2 or 3 times a year. To illustrate this, we hypothesized that sensitivity could be increased by using a number $(2,3$, 4 , or 5) of borescope exams interpreted in parallel (i.e., feet are considered diseased as soon as more than one exam indicates so). We tested this hypothesis using the model for active versus inactive or no DD lesions and allowing for conditional dependence between tests. This would probably represent well how a borescope could be integrated in routine DD monitoring. Cows would be examined regularly (e.g., once a week); whenever active DD lesions were noted, and the cow could be pulled into the trimming chute for better control of the disease. Although the Se for a single borescope exam for diagnosing active DD lesions was estimated at $32 \%$ (95\% CrI: 13,58$)$, the Se after $2,3,4$, or 5 borescope exams would be estimated at $53 \%$ (95\% CrI: 24,83$)$, 68\% (95\% CrI: 34,93$), 78 \%$ (95\% CrI: 43,97 ), and $85 \%$ (95\% CrI: 50, 99), respectively. Thus, 5 borescope exams conducted at weekly intervals, for instance, would lead to similar sensitivity to that of the trimming chute exam.

Finally, the use of a commercial borescope makes it possible to archive images of DD lesions for further use, such as monitoring treatment and control programs and the evolution of the disease, and to train farm workers involved in DD intervention. Images could be transferred remotely for scoring by a more experienced diagnostician. Recently, a study was published on the agreement of DD diagnosis between different experts from different institutions using digital photos (Vanhoudt et al., 2019). The results from this study, coupled with the possibility of increasing the interobserver agreement with training (Relun et al., 2011; Solano et al., 2017), are promising to increase the use of borescopes directly for monitoring and conducting research on DD on dairy farms.

\section{Study Limitations}

This study has some limitations. Only one herd was tested; therefore, the external validity of our experience can be limited. We think that the herd is representative of freestall herds in Québec with a standard milking parlor facility. Because of our 1-population, 2-test design, we relied on prior information, especially when adding extra parameters, such as the conditional dependence between the tests. The accuracy of the borescope was tested in a limited number of animals and under conditions that are strictly connected to the characteristics of the farm itself. We were thus unable to assess the potential impact of some environmental conditions and of cow covariates on borescope accuracy. Milking parlor lighting and characteristics and the position of the cows (oblique parlor vs. other types of positions) are among the covariates that could affect the quality of borescope interpretation and therefore the accuracy of the test.

\section{CONCLUSIONS}

The commercial borescope was convenient and handy and had excellent inter-rater agreement. The Se and Sp of a single borescope exam for diagnosing active DD lesions were estimated at 32 and $91 \%$, respectively. 
The Se of a single borescope exam is thus quite low. In contrast, the Se and Sp of a trimming chute exam were estimated at 91 and $81 \%$, respectively, indicating that routine trimming chute examination should not be considered a gold standard test for DD diagnosis because there is a risk of misclassifying the DD lesions. Finally, the accuracy of a diagnostic strategy based on regular borescope exams for diagnosing active DD lesions was comparable to that of a single trimming chute exam after 5 borescope exams. Further studies may be needed to assess the accuracy of commercial borescopes using different models.

\section{ACKNOWLEDGMENTS}

The authors are grateful to Fermes J. N. Beauchemin Inc. (St-Ours, Québec, Canada) for its willingness to participate to this study, to Adeline Hervé and JeanPhilippe Pelletier (Faculté de Médecine Vétérinaire, Université de Montréal, St-Hyacinthe, Québec, Canada) for their help in data collection and management of data. The authors have not stated any conflicts of interest.

\section{REFERENCES}

Anklam, K., M. Kulow, W. Yamazaki, and D. Döpfer. 2017. Development of real-time PCR and loop-mediated isothermal amplification (LAMP) assays for the differential detection of digital dermatitis associated treponemes. PLoS One 12:e0178349. https://doi .org/10.1371/journal.pone.0178349.

Berry, S. L., D. H. Read, T. R. Famula, A. Mongini, and D. Döpfer. 2012. Long-term observations on the dynamics of bovine digital dermatitis lesions on a California dairy after topical treatment with lincomycin $\mathrm{HCl}$. Vet. J. 193:654-658. https://doi.org/10 $.1016 / j . t v j 1.2012 .06 .048$.

Branscum, A. J., I. A. Gardner, and W. O. Johnson. 2005. Estimation of diagnostic-test sensitivity and specificity through Bayesian modeling. Prev. Vet. Med. 68:145-163. https://doi.org/10.1016/j .prevetmed.2004.12.005.

Cha, E., J. A. Hertl, D. Bar, and Y. T. Grohn. 2010. The cost of different types of lameness in dairy cows calculated by dynamic programming. Prev. Vet. Med. 97:1-8. https://doi.org/10.1016/j .prevetmed.2010.07.011.

Cohen, J. 1968. Weighted kappa: Nominal scale agreement with provision for scaled disagreement or partial credit. Psychol. Bull. 70:213-220. https://doi.org/10.1037/h0026256.

Cramer, G., K. D. Lissemore, C. L. Guard, K. E. Leslie, and D. F. Kelton. 2008. Herd- and cow-level prevalence of foot lesions in Ontario dairy cattle. J. Dairy Sci. 91:3888-3895. https://doi.org/ 10.3168/jds.2008-1135.

Cramer, G., T. Winders, L. Solano, and D. H. Kleinschmit. 2018. Evaluation of agreement among digital dermatitis scoring methods in the milking parlor, pen, and hoof trimming chute. J. Dairy Sci. 101:2406-2414. https://doi.org/10.3168/jds.2017-13755.

Dohoo, I. R., S. W. Martin, and H. Stryhn. 2009. Veterinary Epidemiologic Research. 2nd ed. VER Inc., Charlottetown, PE, Canada.

Döpfer, D., K. Anklam, D. Mikheil, and P. Ladell. 2012. Growth curves and morphology of three Treponema subtypes isolated from digital dermatitis in cattle. Vet. J. 193:685-693. https://doi.org/10.1016/ j.tvjl.2012.06.054.

Döpfer, D., A. A. H. M. ter Huurne, J. L. Cornelisse, A. J. A. M. van Asten, A. Koopmans, F. A. Meijer, Y. H. Schukken, I. Szakall,
W. Klee, and R. B. Bosma. 1997. Histological and bacteriological evaluation of digital dermatitis in cattle, with special reference to spirochaetes and Campylobacter faecalis. Vet. Rec. 140:620-623. https://doi.org/10.1136/vr.140.24.620.

Dutton-Regester, K. J., T. S. Barnes, J. D. Wright, J. I. Alawneh, and A. R. Rabiee. 2018. A systematic review of tests for the detection and diagnosis of foot lesions causing lameness in dairy cows. Prev. Vet. Med. 149:53-66. https://doi.org/10.1016/j.prevetmed.2017.11 .003 .

Evans, N. J., R. D. Murray, and S. D. Carter. 2016. Bovine digital dermatitis: Current concepts from laboratory to farm. Vet. J. 211:3-13. https://doi.org/10.1016/j.tvjl.2015.10.028.

Gomez, A., N. B. Cook, N. D. Bernardoni, J. Rieman, A. F. Dusick, R. Hartshorn, M. T. Socha, D. H. Read, and D. Döpfer. 2012. An experimental infection model to induce digital dermatitis infection in cattle. J. Dairy Sci. 95:1821-1830. https://doi.org/10.3168/jds .2011-4754.

Gomez, A., N. B. Cook, J. Rieman, K. A. Dunbar, K. E. Cooley, M. T. Socha, and D. Döpfer. 2015a. The effect of digital dermatitis on hoof conformation. J. Dairy Sci. 98:927-936. https://doi.org/ $10.3168 /$ jds. 2014-8483.

Gomez, A., N. B. Cook, M. T. Socha, and D. Döpfer. 2015b. Firstlactation performance in cows affected by digital dermatitis during the rearing period. J. Dairy Sci. 98:4487-4498. https://doi.org/10 $.3168 /$ jds.2014-9041.

Jacobs, C., K. Orsel, and H. W. Barkema. 2017. Prevalence of digital dermatitis in young stock in Alberta, Canada, using pen walks. J. Dairy Sci. 100:9234-9244. https://doi.org/10.3168/jds.2017-13044.

Jacobs, C., K. Orsel, S. Mason, and H. W. Barkema. 2018. Comparison of effects of routine topical treatments in the milking parlor on digital dermatitis lesions. J. Dairy Sci. 101:5255-5266. https://doi .org/10.3168/jds.2017-13984.

Jones, G., and W. O. Johnson. 2016. A Bayesian superpopulation approach to inference for finite populations based on imperfect diagnostic outcomes. J. Agric. Biol. Environ. Stat. 21:314-327. https:/ /doi.org/10.1007/s13253-015-0239-9.

Laven, R. A. 1999. The environment and digital dermatitis. Cattle Pract. 7:349-354.

Leeflang, M. M., P. M. Bossuyt, and L. Irwig. 2009. Diagnostic test accuracy may vary with prevalence: implications for evidence-based diagnosis. J. Clin. Epidemiol. 62:5-12. https://doi.org/10.1016/j .jclinepi.2008.04.007.

Leeflang, M. M., A. W. Rutjes, J. B. Reitsma, L. Hooft, and P. M. Bossuyt. 2013. Variation of a test's sensitivity and specificity with disease prevalence. Can. Med. Assoc. J. 185:E537-544.

Manske, T., J. Hultgren, and C. Bergsten. 2002. Topical treatment of digital dermatitis associated with severe heel-horn erosion in a Swedish dairy herd. Prev. Vet. Med. 53:215-231. https://doi.org/ 10.1016/S0167-5877(01)00268-9.

Orsel, K., P. Plummer, J. Shearer, J. De Buck, S. D. Carter, R. Guatteo, and H. W. Barkema. 2018. Missing pieces of the puzzle to effectively control digital dermatitis. Transbound. Emerg. Dis. 65(Suppl. 1):186-198. https://doi.org/10.1111/tbed.12729.

Plummer, P. J., and A. Krull. 2017. Clinical perspectives of digital dermatitis in dairy and beef cattle. Vet. Clin. North Am. Food Anim. Pract. 33:165-181. https://doi.org/10.1016/j.cvfa.2017.02.002.

Quintero, A., and E. Lesaffre. 2018. Comparing hierarchical models via the marginalized deviance information criterion. Stat. Med. 37:2440-2454. https://doi.org/10.1002/sim.7649.

Relun, A., R. Guatteo, P. Roussel, and N. Bareille. 2011. A simple method to score digital dermatitis in dairy cows in the milking parlor. J. Dairy Sci. 94:5424-5434. https://doi.org/10.3168/jds $.2010-4054$.

Relun, A., A. Lehebel, M. Bruggink, N. Bareille, and R. Guatteo. 2013a. Estimation of the relative impact of treatment and herd management practices on prevention of digital dermatitis in French dairy herds. Prev. Vet. Med. 110:558-562. https://doi.org/ 10.1016/j.prevetmed.2012.12.015.

Relun, A., A. Lehebel, A. Chesnin, R. Guatteo, and N. Bareille. 2013b. Association between digital dermatitis lesions and test-day milk 
yield of Holstein cows from 41 French dairy farms. J. Dairy Sci. 96:2190-2200. https://doi.org/10.3168/jds.2012-5934.

Solano, L., H. W. Barkema, C. Jacobs, and K. Orsel. 2017. Validation of the M-stage scoring system for digital dermatitis on dairy cows in the milking parlor. J. Dairy Sci. 100:1592-1603. https:// doi.org/10.3168/jds.2016-11365.

Solano, L., H. W. Barkema, S. Mason, E. A. Pajor, S. J. LeBlanc, and K. Orsel. 2016. Prevalence and distribution of foot lesions in dairy cattle in Alberta, Canada. J. Dairy Sci. 99:6828-6841. https://doi .org/10.3168/jds.2016-10941.

Stokes, J. E., K. A. Leach, D. C. Main, and H. R. Whay. 2012. The reliability of detecting digital dermatitis in the milking parlour. Vet. J. 193:679-684. https://doi.org/10.1016/j.tvj1.2012.06.053.

Thomsen, P. T., I. C. Klaas, and K. Bach. 2008. Short communication: scoring of digital dermatitis during milking as an alternative to scoring in a hoof trimming chute. J. Dairy Sci. 91:4679-4682. https://doi.org/10.3168/jds.2008-1342.

Timsit, E., R. Leguillette, B. J. White, R. L. Larson, and S. Buczinski. 2018. Likelihood ratios: an intuitive tool for incorporating diagnostic test results into decision-making. J. Am. Vet. Med. Assoc. 252:1362-1366. https://doi.org/10.2460/javma.252.11.1362.

Van Hertem, T., Y. Parmet, M. Steensels, E. Maltz, A. Antler, A. A. Schlageter-Tello, C. Lokhorst, C. E. Romanini, S. Viazzi, C. Bahr, D. Berckmans, and I. Halachmi. 2014. The effect of routine hoof trimming on locomotion score, ruminating time, activity, and milk yield of dairy cows. J. Dairy Sci. 97:4852-4863. https://doi.org/10 $.3168 /$ jds.2013-7576.

Vanhoudt, A., D. A. Yang, T. Armstrong, J. N. Huxley, R. A. Laven, A. D. Manning, R. F. Newsome, M. Nielen, T. van Werven, and
N. J. Bell. 2019. Interobserver agreement of digital dermatitis Mscores for photographs of the hind feet of standing dairy cattle. J. Dairy Sci. 102:5466-5474. https://doi.org/10.3168/jds.2018-15644.

Yang, D. A., C. Heuer, R. Laven, W. D. Vink, and R. N. Chesterton. 2017. Estimating the true prevalence of bovine digital dermatitis in Taranaki, New Zealand using a bayesian latent class model. Prev. Vet. Med. 147:158-162. https://doi.org/10.1016/j.prevetmed 2017.09.008.

Yang, D. A., and R. A. Laven. 2019. Detecting bovine digital dermatitis in the milking parlour: To wash or not to wash, a Bayesian superpopulation approach. Vet. J. 247:38-43. https://doi.org/10 $.1016 / j . t v j 1.2019 .02 .011$.

Zinicola, M., F. Lima, S. Lima, V. Machado, M. Gomez, D. Döpfer, C. Guard, and R. Bicalho. 2015. Altered microbiomes in bovine digital dermatitis lesions, and the gut as a pathogen reservoir. PLoS One 10:e0120504. https://doi.org/10.1371/journal.pone.0120504.

\section{ORCIDS}

Sébastien Buczinski ๑ https://orcid.org/0000-0002-8460-4885

Simon Dufour ㄴ https://orcid.org/0000-0001-6418-0424

Jocelyn Dubuc @ (ㄴ https://orcid.org/0000-0003-1013-8372

Jean-Philippe Roy @ https://orcid.org/0000-0002-0444-2303

André Desrochers ํㅏ https://orcid.org/0000-0002-0568-4478 